\title{
Current-induced atomic forces in gated graphene nanoconstrictions
}

\author{
Leitherer, Susanne; Papior, Nick Rübner; Brandbyge, Mads
}

Published in:

Physical Review B

Link to article, DOI:

10.1103/PhysRevB.100.035415

Publication date:

2019

Document Version

Publisher's PDF, also known as Version of record

Link back to DTU Orbit

Citation (APA):

Leitherer, S., Papior, N. R., \& Brandbyge, M. (2019). Current-induced atomic forces in gated graphene nanoconstrictions. Physical Review B, 100(3), [035415]. https://doi.org/10.1103/PhysRevB.100.035415

\section{General rights}

Copyright and moral rights for the publications made accessible in the public portal are retained by the authors and/or other copyright owners and it is a condition of accessing publications that users recognise and abide by the legal requirements associated with these rights.

- Users may download and print one copy of any publication from the public portal for the purpose of private study or research.

- You may not further distribute the material or use it for any profit-making activity or commercial gain

- You may freely distribute the URL identifying the publication in the public portal

If you believe that this document breaches copyright please contact us providing details, and we will remove access to the work immediately and investigate your claim. 


\title{
Current-induced atomic forces in gated graphene nanoconstrictions
}

\author{
S. Leitherer, ${ }^{1, *}$ N. Papior, ${ }^{2}$ and M. Brandbyge ${ }^{1, \dagger}$ \\ ${ }^{1}$ Center for Nanostructured Graphene, Department of Physics, Technical University of Denmark, DK-2800 Kongens Lyngby, Denmark \\ ${ }^{2}$ Department of Applied Mathematics and Computer Science, Technical University of Denmark, DK-2800 Kongens Lyngby, Denmark
}

(Received 1 May 2019; published 12 July 2019)

\begin{abstract}
Electronic current densities can reach extreme values in highly conducting nanostructures where constrictions limit current. For bias voltages on the $1 \mathrm{~V}$ scale, the highly nonequilibrium situation can influence the electronic density between atoms, leading to significant interatomic forces of the order of $1 \mathrm{nN}$. An easy interpretation of the nonequilibrium forces is currently not available, to our knowledge. In this work, we present an $a b$ initio study based on density functional theory of bias-induced atomic forces in gated graphene nanoconstrictions consisting of junctions between graphene electrodes and graphene nanoribbons in the presence of current. We find that current-induced bond forces and bond charges are correlated, while bond forces are not simply correlated to bond currents. We discuss, in particular, how the forces are related to induced charges and the electrostatic potential profile (voltage drop) across the junctions. For long current-carrying junctions we may separate the junction into a part with a voltage drop, and a part without voltage drop. The latter situation can be compared to a nanoribbon in the presence of current using an ideal ballistic velocity-dependent occupation function. This shows how the combination of voltage drop and current give rise to the strongest current-induced forces in nanostructures.
\end{abstract}

DOI: 10.1103/PhysRevB.100.035415

\section{INTRODUCTION}

The current densities in nanoscale, ballistic conductors can reach extreme values compared to macroscopic Ohmic conductors. For example, the breakdown voltages of atomic chains of $\mathrm{Au}$ are beyond $1 \mathrm{~V}$ corresponding to a currentdensity [1] on the order of $10^{10} \mathrm{~A} / \mathrm{cm}^{2}$, and the currentcarrying capacity of narrow graphene conductors can reach almost $10^{9} \mathrm{~A} / \mathrm{cm}^{2}$ before breakdown [2]. From a technological point of view the nanoregime poses challenges in terms of stability and reproducibility, since in this extreme scaling limit the position of a few atoms control the device operation.

On the other hand, atomic control of the structure by external driving forces offers an enormous potential for further downscaling. For example, it has been demonstrated in experiments how the current/field may be used to toggle switch atomic-scale contacts between different conductance states corresponding to different atomic configurations of metallic nanocontacts [3].

In this paper we will concentrate on another important example, namely graphene nanostructures, which are now being created and changed using high applied voltages and consequently electrical current. Due to its excellent electrical and mechanical properties, graphene is a promising material for two-dimensional (2D) nanoelectronic applications [4]. Socalled "electroburning" has been employed in experiments to fabricate nanogaps between graphene electrodes [5]. These electrodes of single or few-layer graphene have in some cases subsequently been bridged by single molecules [6-9]. Using similar techniques, the fabrication of electrically switchable graphene break junctions has been reported [10,11]. Electron microscopy allows for structural, atomic-scale studies

\footnotetext{
*Corresponding author: slei@dtu.dk

${ }^{\dagger}$ Corresponding author: mabr@dtu.dk
}

of graphene structures in the presence of high current and applied voltage [12]. It has been seen how the structure of edges are changed by the current/voltage [13] or how layers fuse [14]. Current-induced motion/cleaning of adsorbed species on graphene has also been investigated [2]. Structural dynamics and the relation to the conductance of graphene nanoribbons have been studied in Refs. $[15,16]$. Under high bias and current density a number of different, possibly intertwined, effects play crucial roles for the atomic configuration, such as motion driven by locally induced fields, Joule heating and temperature gradients, as well as current-induced forces due to a steady momentum transfer from electronic current to ions [17-19]. Common for these structures and effects in graphene nanostructures is that the electrons may to a large degree be in the ballistic quantum transport regime, as seen, e.g., by the appearance of interference phenomena [5,20,21]. Experiments performed at high voltage bias on a bilayer constriction show an uniaxial lattice expansion of more than $5 \%$ at a current density on the order of $10^{9} \mathrm{~A} / \mathrm{cm}^{2}$ before breaking [22].

The understanding of the role of voltage and currents in such systems and processes are still rudimentary. We consider here a simple, narrow graphene ribbon system using first principles calculations based on density functional theory combined with Non-equilibrium Greens functions (DFT-NEGF). We have previously studied the electron-phonon interaction in transport and the voltage drop dependence on gating in this system [23-25]. In this paper we consider the currentinduced forces in the presence of steady-state electronic current, and analyze these in terms of the changes in electronic distributions.

Our DFT-NEGF calculations, presented in the first part of this work, return forces for systems that are defected in the sense of having a scattering region. However, for ballistic bulk systems one could imagine a current flowing that is far 


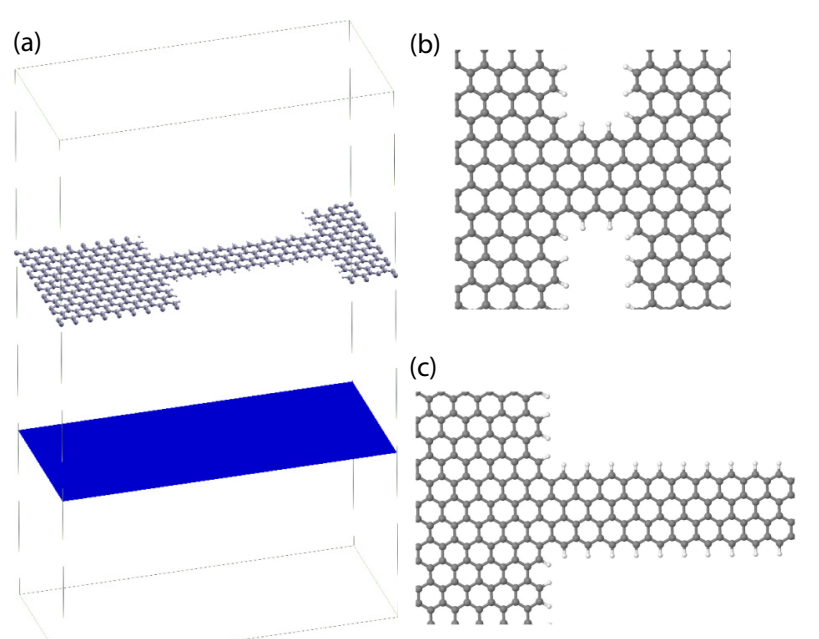

FIG. 1. Graphene nanoconstrictions with electrostatic bottom gate. (a) Represents the unit cell of a generic constriction, with the blue square indicating the position and shape of the electrostatic gate. Edge atoms of the graphene are saturated by hydrogen in order to avoid dangling bonds. (b) Short constriction and (c) semi-infinite GNR constrictions considered in this work.

from any scattering potential, and approximately behaves as though states are occupied depending on their velocity. We present this approach and compare it to the DFT-NEGF forces in Sec. III B 2.

\section{SETUP AND METHOD}

The systems we investigate are constrictions consisting of graphene nanoribbons (GNRs) of varying lengths, placed between graphene electrodes (cf. Fig. 1). In addition, the junctions are electrostatically gated. Their geometries are relaxed at zero bias using the SIESTA package and their properties are studied at finite bias using the nonequilibrium electronic transport package TRANSIESTA. Computational details are described in Sec. V.

We apply the field-effect gate model of Ref. [25]. A charged plane is placed at $15 \AA$ underneath the graphene constriction. The plane carries a charge density of $n=g \times$ $10^{13} e^{-} / \mathrm{cm}^{2}$, where $g$ defines the gating levels, with $g<$ $0 / g>0$ referring to $n / p$ doping. Placing the electrostatic gate allows for a tuning of the conductance of the junction, while on the other hand, the position of the voltage drop in the constriction can be controlled [25]. Thus we explicitly include the role of the gate-induced carriers on the screening properties and potential profile.

We focus on two distinct geometries, shown in Figs. 1(b) and 1(c): The first (b) is a graphene constriction with a very short GNR, and the second junction (c), consists of a large region of pristine graphene connected to a semi-infinite GNR. The results are presented in Sec. III A and Sec. III B, respectively.

Since our aim here is to study generic features of the local current and potential drop, and the relation to inter-atomic forces, we neglect the role of spin-polarization at the zig-zag edges [26]. (a)

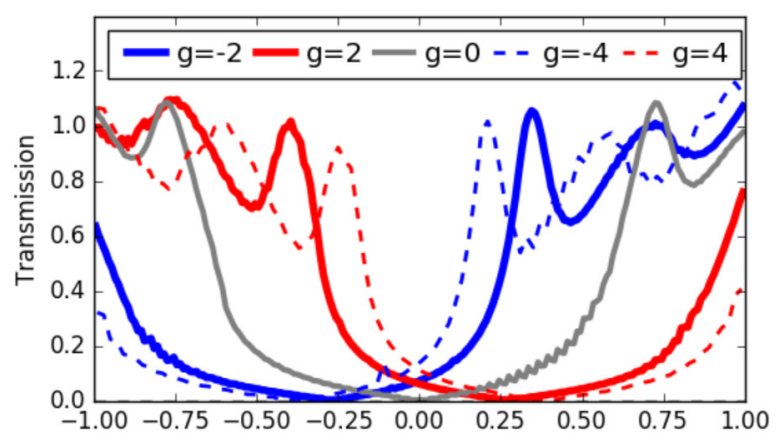

(b)

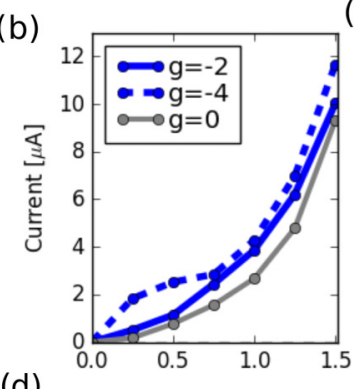

(c) Energy [ev]
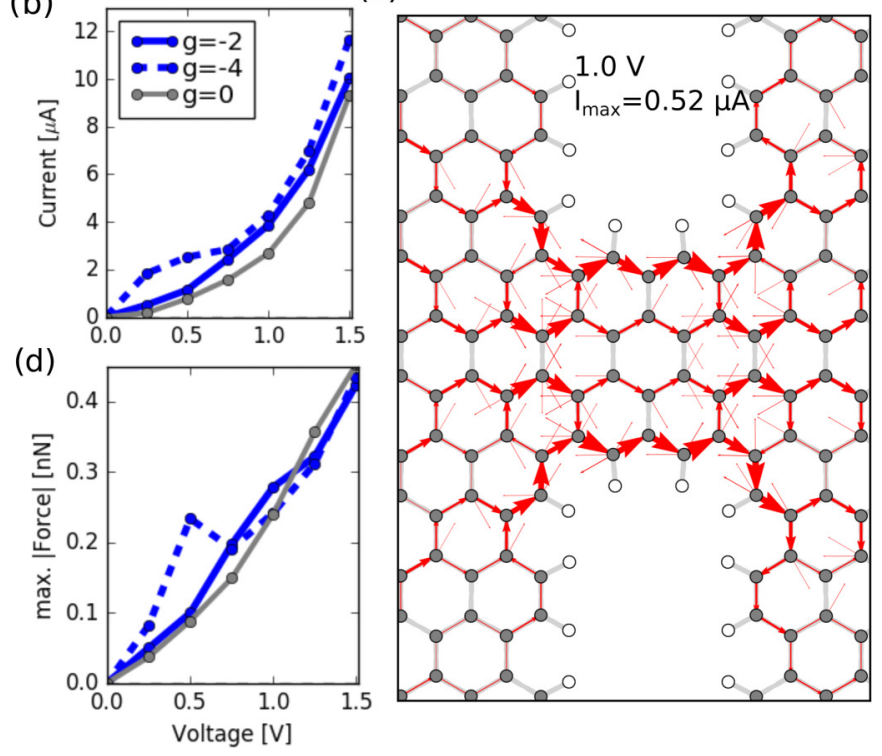

FIG. 2. (a) Zero-bias transmission for different gate charges $g$ (gray without gate), (b) total current as a function of bias voltage for selected gate charges, (c) real-space bond currents at $1 \mathrm{~V}, g=-2$, and (d) maximal absolute force over bias voltage for different gate charges.

\section{RESULTS AND DISCUSSION}

\section{A. Short constriction}

We first consider the left-right symmetric graphene nanojunction with a short GNR [cf. Fig. 1(b)]. We employ periodic boundary conditions in the direction transverse to the constriction with a corresponding $k$-point sampling.

\section{Conductance properties}

In Figs. 2(a)-2(c), we discuss the transport properties of the junction, in particular the transmission probability, total currents, and real-space "bond currents". In the following we implicitly assume $k$-dependence. Thereby, the transmission through the constriction is calculated from [27]

$$
T(E, V)=\operatorname{Tr}\left[\mathbf{G} \boldsymbol{\Gamma}_{L} \mathbf{G}^{\dagger} \boldsymbol{\Gamma}_{R}\right],
$$

where $\mathbf{G}=[(E+i \eta) \mathbf{S}-\mathbf{H}-\mathbf{\Sigma}]^{-1}$ is the nonequilibrium retarded Green's function with device Hamiltonian $\mathbf{H}$, overlap $\mathbf{S}$ and self-energies $\boldsymbol{\Sigma}=\sum_{\alpha=L, R} \boldsymbol{\Sigma}^{\alpha}$, and $\boldsymbol{\Gamma}^{\alpha}=i\left(\boldsymbol{\Sigma}^{\alpha \dagger}-\boldsymbol{\Sigma}^{\alpha}\right)$. The total current is given by [28]

$$
I(V)=\frac{2 e}{h} \int T(E, V)\left[f_{L}(E, V)-f_{R}(E, V)\right] \mathrm{d} E,
$$


with $f_{L / R}$ being the Fermi distributions in the electrodes, where the chemical potentials at finite bias are shifted according to $\mu_{L / R}=E_{F} \pm e V / 2$.

Figure 2(a) shows the zero-bias transmission for different values of the gating parameter $g$. Gating leads to a doping of the junction, i.e., the charge-neutrality point in the density of states (DOS) is shifted relative to its position at $g=0$. Accordingly the transmission is shifted further into the conductance window with $g$. This results in a higher conductivity especially at small bias, cf. total current in Fig. 2(b). In the high bias regime the current is not significantly enhanced by the gating, because the transmission at high energies is nearly 1 .

A spatial distribution of the current flowing through the junction can be obtained by calculating bond currents [29-31]. The energy-dependent spectral bond currents from atom $n$ to $m$ are defined as

$$
\partial J_{n m}(E, V)=\frac{2 e}{\hbar} \sum_{\mu, \nu} \operatorname{Im}\left\{\mathbf{A}_{\mu \nu}^{\alpha}(E, V)[\mathbf{H}(V)-E \mathbf{S}]_{\nu \mu}\right\}
$$

where $\alpha=L, R$ refers to the electrode, $\nu \in n$ and $\mu \in m$ are orbital indices, and the spectral function is given by

$$
\mathbf{A}^{\alpha}(E, V)=\mathbf{G} \boldsymbol{\Gamma}^{\alpha} \mathbf{G}^{\dagger} \text {. }
$$

The bond current is obtained by integrating Eq. (3) over the Fermi window, defined by $f_{L}-f_{R}$ at the corresponding bias:

$$
J_{n m}(V)=\frac{1}{2 \pi} \int \partial J_{n m}(E, V)\left[f_{L}(E, V)-f_{R}(E, V)\right] \mathrm{d} E .
$$

In Fig. 2(c) the bond currents at a bias of $1 \mathrm{~V}$ are shown. The highest current density appears at the entrance to the constriction and along the edge atoms in the constriction. In the pristine graphene, bond currents obtain smaller values and spread out across the lattice. They obey the law of particle conservation, i.e., through any section dividing the left and right part their total sum is conserved. The current pattern exhibits a somewhat left-right/top-bottom symmetry, which obviously stems from the junction symmetry.

We want to study the interatomic forces in the graphene constriction, which are induced when a finite bias voltage is applied. These forces are calculated in the Born-Oppenheimer approximation from the nonequilibrium electron density defined by the density matrix $\mathbf{D}$, which we obtain from the DFT-NEGF formalism [32]. In particular, the force acting on atom $n$ with coordinate $\vec{R}_{n}$ is given through the force operator $\overrightarrow{\mathbf{F}}_{n}$ and the density operator $\mathbf{D}$ via

$$
\vec{F}_{n}=\operatorname{Tr}\left[\overrightarrow{\mathbf{F}}_{n} \mathbf{D}\right]=-\operatorname{Tr}\left[\frac{\partial \mathbf{H}}{\partial \vec{R}_{n}} \mathbf{D}\right],
$$

and the nonequilibrium density operator,

$$
\mathbf{D}=\int\left[\mathbf{A}^{L}(E, V) f_{L}(E, V)+\mathbf{A}^{R}(E, V) f_{R}(E, V)\right] \mathrm{d} E .
$$

In Fig. 2(d), we plot the maximum absolute force induced by the nonequilibrium between all atoms in the short GNR constriction, depending on the gate parameter and the bias voltage (for a spatial distribution of the forces, see below). The maximum force is seen to increase with voltage roughly following the current, where as both are more weakly influenced by the gate parameter. We find forces of $\sim 0.2 \mathrm{nN}$ at $1 \mathrm{~V}$.

Theoretical models were compared to tunnel-to-contact experiments of atomic point contacts in order to explicitly (a)

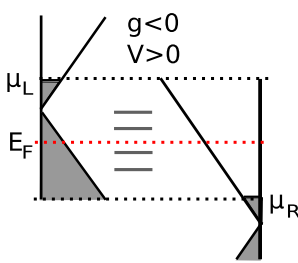

(d)

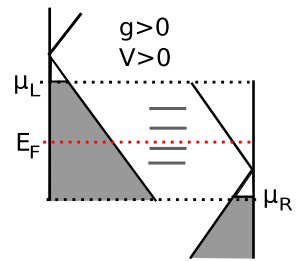

(b)

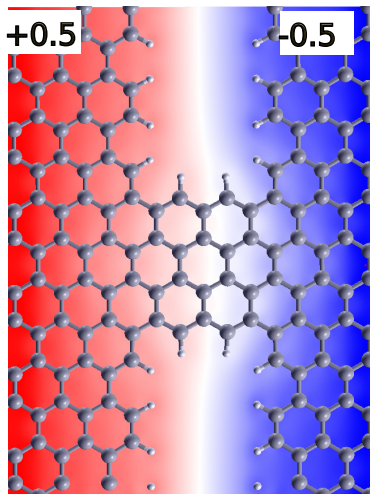

(c)

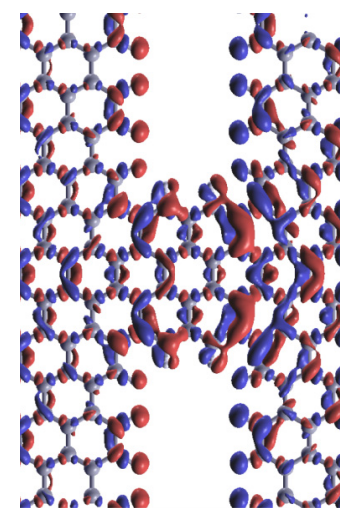

(e)

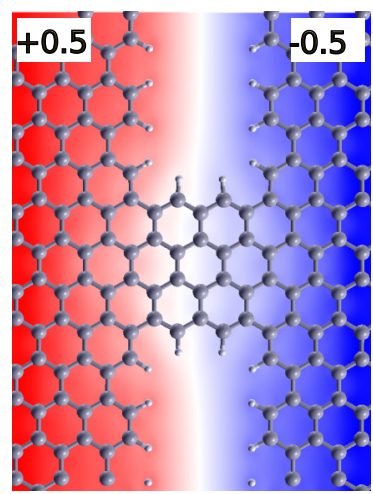

(f)

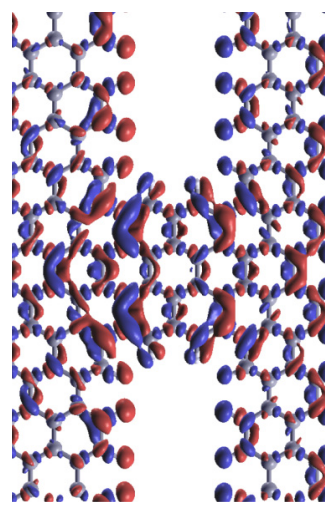

FIG. 3. (a) Energy scheme of the electrode DOS for negative $g$ and positive bias voltage, (b) electrostatic potential profile, and (c) bias-induced charge redistribution at $1 \mathrm{~V}$ for $g=-2$. (d), (e), (f) DOS, potential, and charges at $1 \mathrm{~V}$ for $g=+2$.

relate the conductance to the atomic forces at low bias $\sim 1 \mathrm{mV}$, as for example presented in Ref. [33]. Below, we will present a detailed analysis of the forces and compare these to the local current and potential drop at the higher voltages.

\section{Potential drop and finite bias charge redistribution}

At finite bias, the chemical potential in the electrodes is symmetrically shifted and an electrical field between the electrodes exists across the junction, resulting in a rearrangement of charge. We present in Fig. 3(a) the schematic picture of the electrode DOS and the energy levels of the junction at finite bias for $g=-2$, in Fig. 3(b) the electrostatic potential landscape $\Phi(1 \mathrm{~V}, g=-2)-\Phi(0 \mathrm{~V}, g=-2)$, and in Fig. 3(c) the induced charge $\rho(1 \mathrm{~V},-2)-\rho(0 \mathrm{~V},-2)$ in the short GNR constriction. In Figs. 3(d)-3(f) we present the same analysis for $g=+2$.

The energy scheme, Figs. 3(a) and 3(d), illustrates how the nonsymmetric coupling is induced via the electrode having the largest DOS in the bias window. This results in an electrostatic potential pinning of the constriction. In Fig. 3(b) it pins to the right electrode presenting the larger DOS in the voltage 
(a)

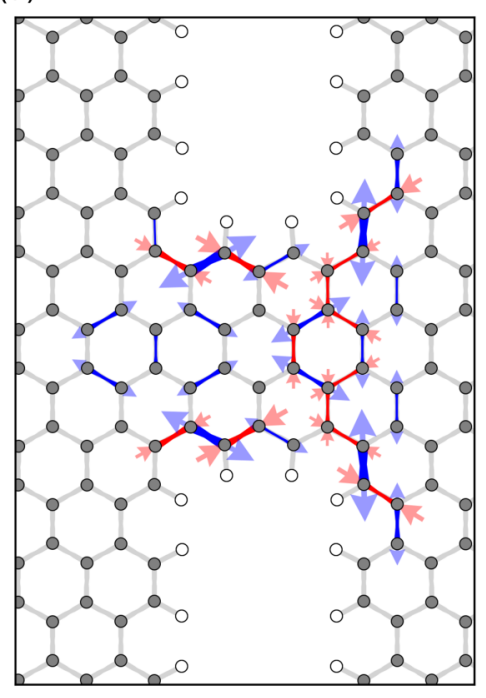

(b)

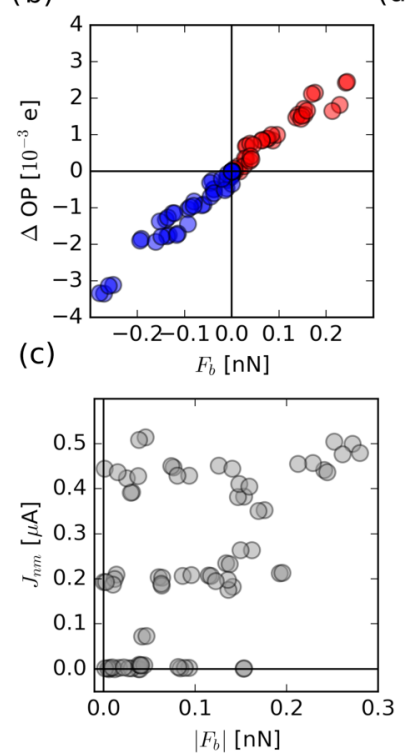

(d)

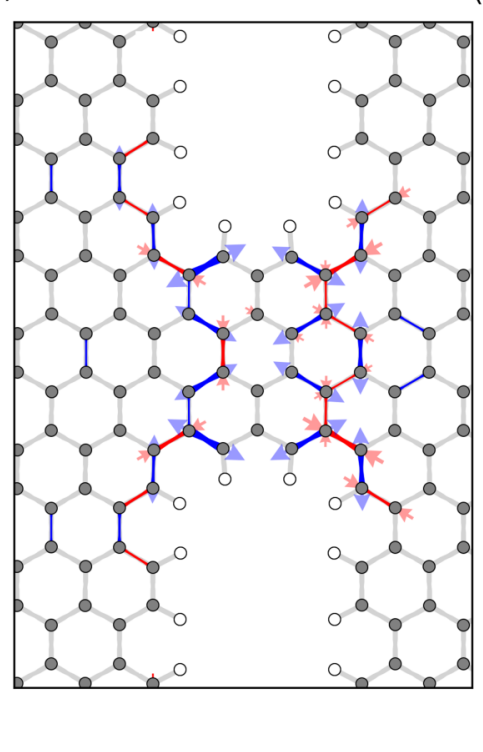

(e)
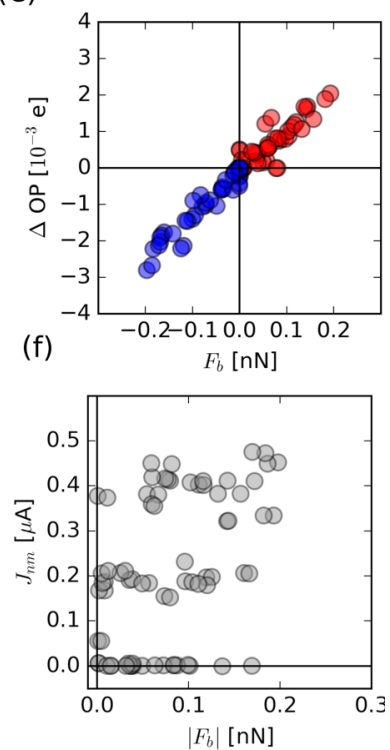

FIG. 4. (a) Induced bond forces, $F_{b}$, and change in overlap population $\Delta \mathrm{OP}$ at $1.0 \mathrm{~V}, g=-2$. The forces $F_{b}$ are shown as vectors in light red and blue. Force vectors are pointing inwards (outwards) to indicate bond compressing (stretching), while the vector thickness corresponds to the force strength. The change in OP is depicted as in-/decreasing density along the bond, in red for positive and blue for negative $\Delta \mathrm{OP}$. Induced forces/charges below a cutoff of $\left|F_{b}\right|=0.004 \mathrm{nN} / \Delta \mathrm{OP}=1 \times 10^{-4}$ e are set to zero. (b) Correlation between bond forces $F_{b}$ and $\Delta \mathrm{OP},(\mathrm{c})$ correlation between bond forces $\left|F_{b}\right|$ and bond currents $J_{n m}$, (d), (e), (f) equivalent pictures for $g=+2$.

window, and opposite for the case in Fig. 3(e), see Ref. [25] for details. Such relative changes in the electrostatic potential also results in a different charge redistribution. In Figs. 3(c) and 3(f) we show how the charge redistribution is highest at the interface of the potential drop.

Our analysis shows that forces are highly correlated with such charge redistributions and in the following we will outline simple relations between the charge redistributions and forces.

\section{Bond forces and overlap population}

To simplify the representation of the forces, Eq. (6), we project them onto the atomic bonds. These bond forces are defined as the difference of the forces on atom $n$ and $m$, projected onto the bond vector $\vec{r}_{n m}=\vec{r}_{n}-\vec{r}_{m}$ :

$$
\vec{F}_{b, n m}=\frac{\left(\vec{F}_{m}-\vec{F}_{n}\right) \cdot \vec{r}_{n m}}{\left|\vec{r}_{n m}\right|}
$$

With this definition, positive (negative) bond forces can be interpreted as compressive (repulsive). Note that our structures are relaxed at zero bias, thus $F_{b}$ refers to the bias induced forces.

Figure 4(a) depicts induced bond forces at $1 \mathrm{~V}$ for $g=2$, and in Fig. 4(d) for $g=-2$. Compressive (repulsive) bond forces are shown as arrows in light red (light blue). We draw the force arrows at both atoms of a bond to indicate if the force is stretching or compressing the bond.

In Figs. 4(a) and 4(d) we also show how we can relate the forces to the charge redistribution in the junction. In particular, we have calculated the amount of charge in the bonds, also termed overlap population (OP), similar to the analysis in Ref. [34]. This approach is based on interpreting the bond population as a measure of the bond strength [35]. The OP is given by a sum over atomic orbitals $(i, j)$ belonging to the atoms $n, m$,

$$
\mathrm{OP}=\sum_{\alpha=L, R} \sum_{\substack{i \in n \\ j \in m}} \mathbf{O}_{i j}^{\alpha}
$$

with

$$
\mathbf{O}_{i j}^{\alpha}=\mathbf{S}_{i j} \int \mathrm{d} E \mathbf{A}_{i j}^{\alpha}(E) f_{\alpha}\left(E-\mu_{\alpha}\right),
$$

and the spectral function $\mathbf{A}^{\alpha}$, where $\alpha=L, R$, since it has contributions from left and right-originating states. To obtain the bias-induced bond charge, we calculate the change in overlap population with bias, $\Delta \mathrm{OP}=\mathrm{OP}(V)-\mathrm{OP}(0)$.

In Figs. 4(a) and 4(d), the nonzero $\Delta \mathrm{OP}$ are depicted as density along the bond; in particular the line thickness corresponds to $|\Delta \mathrm{OP}|$ and red (blue) indicates if it has positive (negative) sign. We find that the bond forces and the change in overlap population are clearly correlated. An increase (decrease) of charge in the bond corresponds to a positive (negative) bond force, corresponding to bond elongation compression (elongation). This correlation between bond force and population is also revealed by the scatter plots in Fig. 4(b) for $g=2$ (and Fig. 4(e) for $g=-2$ ).

Similarly to the bond forces, we plot the bond currents in Fig. 2(c). While the bond currents show a left-right symmetry, this symmetry is fully absent in the bond forces. As shown in Figs. 4(c) and 4(f), the bond current and force strength do not clearly correlate. This suggests that even though certain atoms experience a high current density it is not necessarily reflected in forces acting on it, or at least its effect is minor compared to other effects. We note that recent work calculating the current-induced forces in graphene nanoribbons based 
(a)
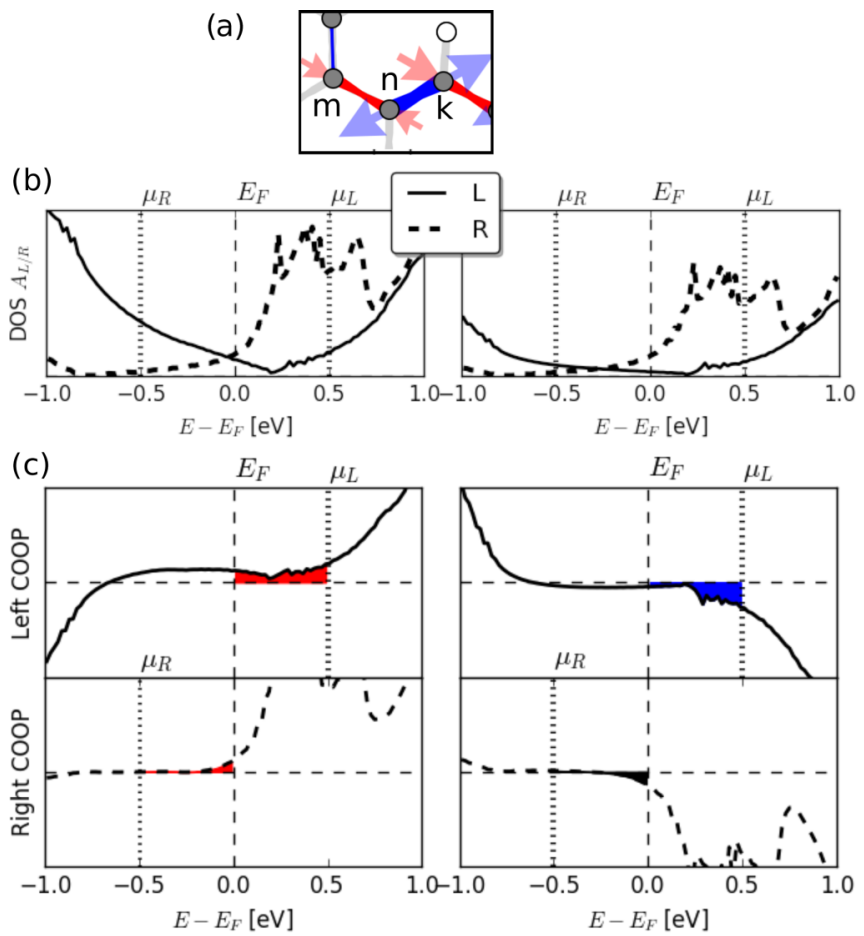

FIG. 5. (a) Chemical bond of atom $m$ and $n$ with compressive bond force (red), and bond of atom $n$ and $k$ with repulsive bond force (blue) at $1 \mathrm{~V}$ and $g=-2$. (b) DOS of left-/right-incoming states, $\mathbf{A}^{L}$ and $\mathbf{A}^{R}$, on atoms $m$ and $n$ (left panel) and atoms $n$ and $k$ (right panel) at $1 \mathrm{~V}$. States in $\mathbf{A}^{L}\left(\mathbf{A}^{R}\right)$ below $\mu_{L}=0.5 \mathrm{eV}$ $\left(\mu_{R}=-0.5 \mathrm{eV}\right)$ are occupied. (c) COOP analysis for bond $m-n$ (left panels) and bond $n-k$ (right panels). Bond $m-n$ is strengthened by current, as at $1 \mathrm{~V}$ bonding states get populated in $\mathbf{A}^{L}$ (red shaded area in left COOP). The DOS that is depleted in $\mathbf{A}_{R}$ is small. Bond $n-k$ is weakened by current, as antibonding states get filled (blue shaded area).

on single-orbital tight-binding model found a correlation between the local currents and bond forces [36]. But this clearly will depend on the level of description of the connection to electrodes and the associated potential drop.

To get further insight into the bias-induced bond populations, we analyze the crystal orbital overlap population (COOP) curve [37], which is the energy-resolved overlap population. For the bond between atoms $n$ and $m$, the COOP is defined as

$$
\operatorname{COOP}(E)=2 \sum_{i \in n, j \in m} \mathbf{S}_{i j} \mathbf{A}_{i j}^{\alpha}(E),
$$

with $\alpha=L, R$ referring to left- and right-incoming states. The sign of the COOP curve determines whether the states contributing to the bond have bonding (positive) or antibonding (negative) character [37]. Therefore filling of bonding/depletion of antibonding states will lead to a strengthening of the bond force, and vice versa. Note that integrating the COOP (weighted by the Fermi distribution) gives the $\mathrm{OP}$.

In Fig. 5 we present this analysis for two bonds in the junction of Fig. 4(a), which experience a high bond force: One bond that is compressed, $m-n$, and one that is stretched, (a)

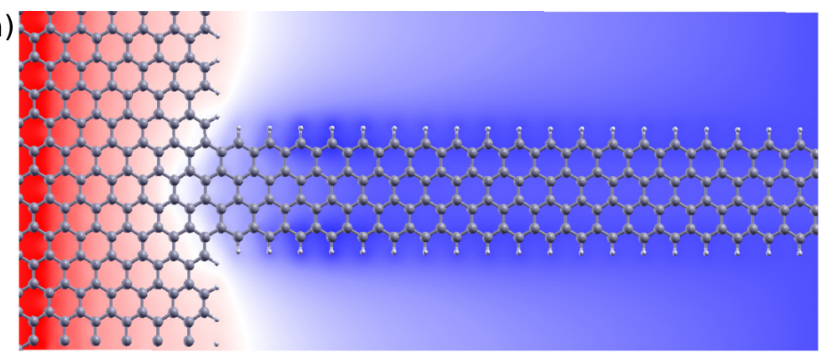

(b)

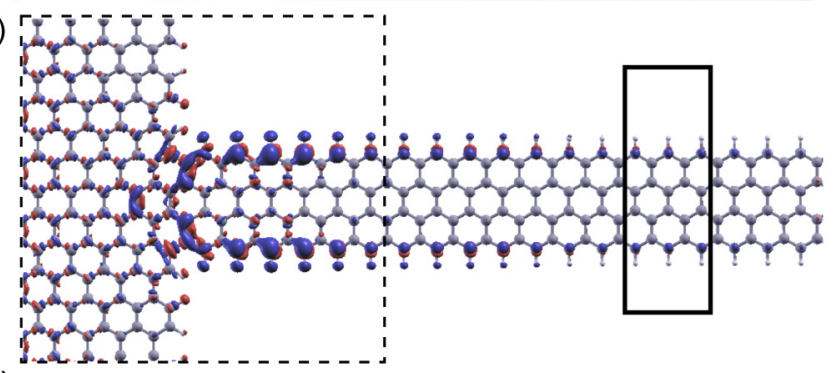

(c)

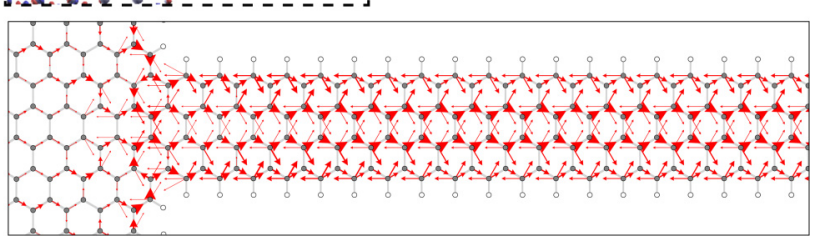

FIG. 6. (a) Electrostatic potential profile (b) bias-induced charge redistribution, and (c) bond currents in the wide-narrow GNR constriction at $0.75 \mathrm{~V}, g=-2$.

$n-k$, under the influence of the current [cf. Fig. 5(a)]. The DOS of left- and right-incoming states at $1 \mathrm{~V}$, shown in Fig. 5(b), is similar for atoms $m-n$ (left panel) and atoms $n-k$ (right panel). However, for atoms $m-n$, the states that are energetically located in the conductance window have bonding character, as indicated by a positive COOP [left panels in Fig. 5(c)], while on atoms $n-k$ they are antibonding [right panels in Fig. 5(c)]. Mostly relevant are states in $\mathbf{A}^{L}$, since those get filled by shifting $\mu_{L}$ up, while the overlap population of right-incoming states (bottom panels) does not change significantly by the downshifting of $\mu_{R}$. The positive $\Delta \mathrm{OP}$ for the bond $m-n$ can be traced back to an increased filling of bonding states, while on atoms $n-k$ antibonding states become occupied, resulting in a negative $\Delta \mathrm{OP}$.

\section{B. Wide-narrow GNR constriction}

In order to study in more detail the influence of the potential profile on the forces, we consider a wide-narrow constriction, where the right electrode is a semi-infinite GNR [cf. Fig. 1(c)]. We focus on a negative doping of $g=-2$ and positive bias voltages.

\section{Potential drop, charge redistribution, bond currents, and forces at nonequilibrium}

In longer GNR constrictions, a pinning of the potential to one of the electrodes can be achieved, leading to a very localized potential drop at the transition between the GNR and the graphene. In Fig. 6(a) we show the potential profile at positive bias (here $0.75 \mathrm{~V}$ ) in a graphene constriction with 


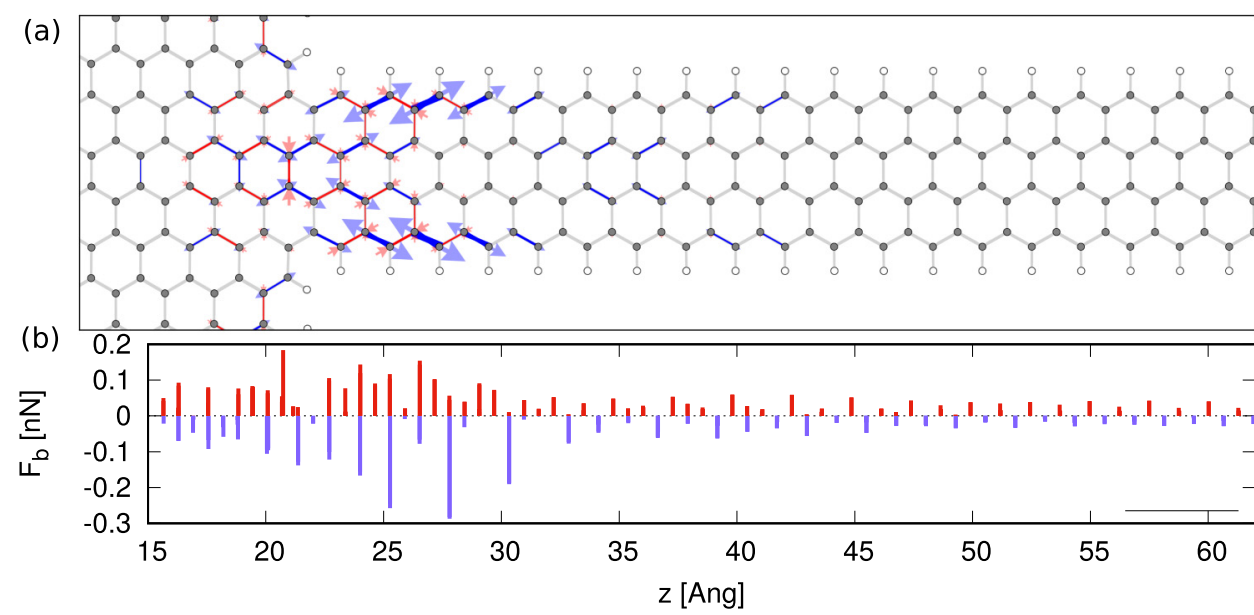

(c)

FIG. 7. (a) Distribution of induced bond forces $F_{b}$ and $\Delta \mathrm{OP}$ in the GNR constriction, (b) maximal values of $F_{b}$ along the transport direction $z$, (c) correlation between $F_{b}$ and $\Delta \mathrm{OP}$, at $0.75 \mathrm{~V}, g=-2$.

a horizontally extended GNR. For a detailed discussion of the bias-dependence of the voltage drop in very similar GNR constrictions, we refer to Ref. [25].

In Fig. 6(b), we show the the bias-induced charge density. We find that the largest amount of charge is accumulated near the potential drop. This is due to the fact that at finite bias, reflection of incoming channels takes place at the scatterer, i.e., the constriction entrance where the potential drop is located. These scattering processes induce Landauer dipoles in this region [38]. Farther away from the potential drop, we find a smaller amount of induced charge density in the GNR, which converges at longer distances. As the states closest to $E_{F}$ are edge states, this charge is mainly localized on the zigzag edges of the GNR.

Figure 6(c) depicts bond currents through the extended GNR constriction. Due to particle conservation, the bond currents are of the same size all along the GNR. Note that there is little to no correspondence between the charge redistribution and bond currents. In a similar way as the charge density profile, the nonequilibrium forces are maximal in the region of the potential drop. This is illustrated in Fig. 7(a), where we show the change in bond forces and overlap population. Again, we find compressive/repulsive forces for bonds where a large amount of bond charge is induced/depleted [cf. Fig. 7(c)]. The maximum/minimum forces along the transport direction in the junction are depicted in Fig. 7(b).

For a detailed analysis of the forces, two regions can be distinguished in the junction: One is the region of the wide-narrow transition [dotted square in Fig. 6(b)], where the potential drop is located. Here we find the largest forces with maximum strength of $\left|F_{b}\right| \leqslant 0.28 \mathrm{nN}$. In this region there are contributions to the forces from the reflected charge density as well as from the density of transmitting channels, beyond the usual electrostatic forces.

Deeper in the GNR, the forces and bond populations become significantly smaller $\left(\left|F_{b}\right| \leqslant 0.05 \mathrm{nN}\right)$ and reach a periodic pattern. In this region [bold square in Fig. 6(b)], the electrostatic potential profile is very flat and nearly equivalent to the right electrode chemical potential, $\mu_{R}$. Thus, the forces in this region are not related to a potential drop, but are ideally solely originating from the flow of current. The correspondence between induced bond forces and $\triangle \mathrm{OP}$ is still given, with the accumulated charge coming from the current in the occupied, transmitted conductance channels.

\section{Forces without voltage drop}

In the extended GNR constriction, we have studied forces in the region away from the resistance-dipole or voltage-drop [bold square in Fig. 6(b)], where a flat potential profile has established. In this region we will not have an electrostatic potential associated with the nonequilibrium, however, we still have a current. This allows for a comparison with a perfectly ballistic bulk system, where a current flows without the electrical field due to the potential drop. One may consider this in an idealized picture of a homogeneous, infinite, periodic nanoribbon where left-moving and right-moving states are occupied differently such that there is a current (but not a field) present in the system. In the simplest model one may consider left-moving and right-moving states filled according to Fermi distributions with different chemical potentials. This approach will of course not include the effect of how the current is fed into the system, e.g., via an interface to pristine graphene with a corresponding filtering by the transmission function.

The simple assumptions allow for the use of the standard periodic boundary conditions and a Bloch band description. Specifically, we may employ a bulk-like calculation scheme where states are occupied according to their band velocity,

$$
v_{n k}=\frac{1}{\hbar} \frac{\partial \varepsilon_{n}(k)}{\partial k},
$$

where $n$ is the band index. The idealized, ballistic occupation function corresponds to a situation where current is fed into the nanoribbon from ideal electrodes without any scattering in the voltage window. This is of course idealized and will overestimate the current. The nonequilibrium distribution function relative to $f_{R}$ is,

$$
\delta f(n k)=\Theta\left(v_{n k} \cdot \hat{\mathbf{e}}\right)\left[f_{L}\left(\varepsilon_{n}(k), V\right)-f_{R}\left(\varepsilon_{n}(k), V\right)\right],
$$

where $\Theta$ is the Heaviside step function and $\hat{\mathbf{e}}$ is the direction of the external bias driving the current. The chemical 


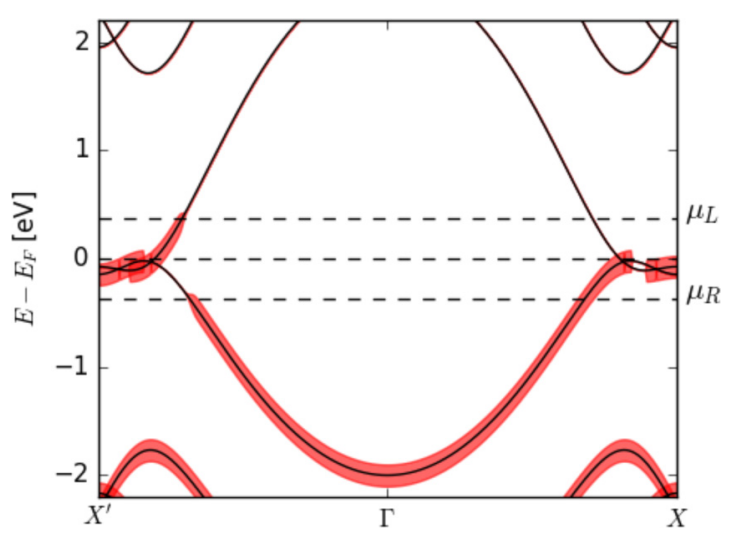

FIG. 8. Bulk-bias applied to GNR. Shown is the band structure, where a positive bias of $0.75 \mathrm{~V}$ along the GNR direction changes the occupation of right-moving states with positive velocity such that states are filled up to $\mu_{L}=e V / 2$. Similarly are the left moving states with negative velocity emptied above $\mu_{R}=-e V / 2$. Filled bands are indicated in red.

potentials for right- and left-movers are $\mu_{L}=E_{F}+e V / 2$ and $\mu_{R}=E_{F}-e V / 2$ with $V$ being the applied voltage. The quasiFermi level, $E_{F}$, is determined in the self-consistent DFT cycle such that the charge is neutral in the unit cell. We will denote this type of calculations as ballistic-bulk calculations.

We have performed a ballistic-bulk calculation for the GNR for $g=-2$. Figure 8 illustrates the filling of bands in the GNR at a bulk bias of $0.75 \mathrm{~V}$. In Fig. 9, we compare the DFT-NEGF forces in the constriction far away from the potential drop with the forces from the ballistic-bulk calculation. The cutout in Fig. 9(a) corresponds to the bold (a)

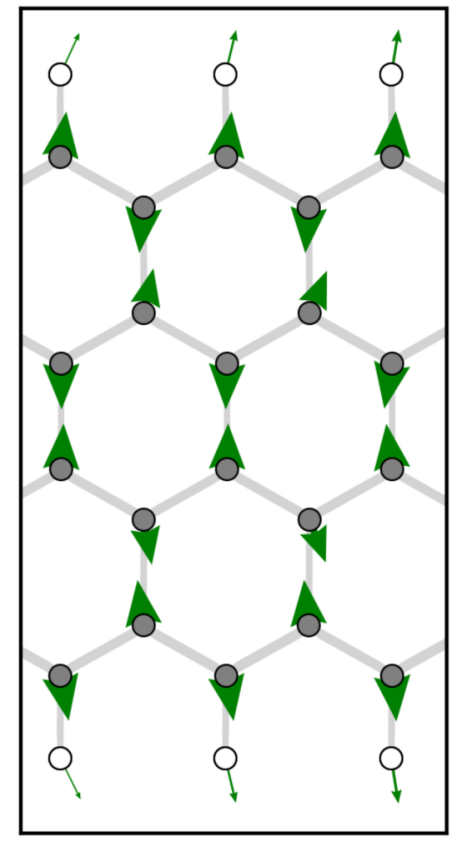

(b)

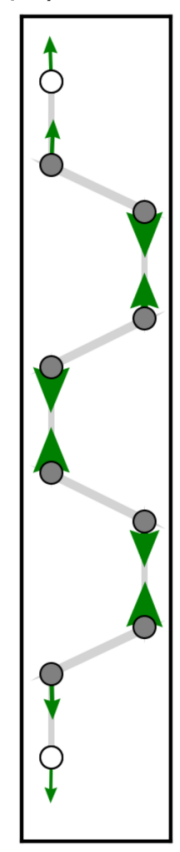

(c)

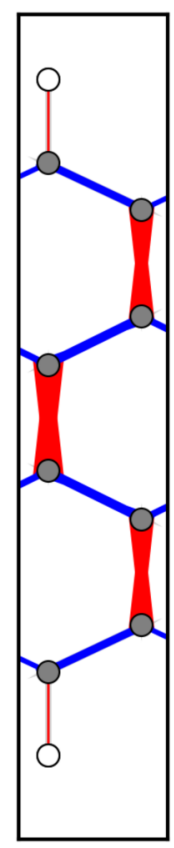

FIG. 9. (a) Force pattern far away from the potential drop at $0.75 \mathrm{~V}$ from TRANSIESTA calculation, (b) forces, and (c) $\triangle \mathrm{OP}$ from bulk calculation at $0.75 \mathrm{~V}$. square in Fig. 6(b), while Fig. 9(b) shows the unit cell of the ballistic-bulk calculation. We recover a very similar force pattern for both calculations, with forces only perpendicular to the transport direction. In both setups, the inner atoms of the GNR are contracting, while the edge atoms and hydrogen are slightly pushed outwards. The correlation between bond forces and induced bond populations is also revealed in the ballistic-bulk calculation, cf. Fig. 9(c). Due to the symmetry of the single unit cell in the bulk calculation, it returns symmetric forces. The DFT forces show some deviations, as Fig. 9(a) is taken out from the large junction. Also, they are lower in magnitude compared to the bulk forces. The maximum bulk forces in Fig. 9(b) are $0.2 \mathrm{nN}$ for $0.75 \mathrm{~V}$, comparable to the forces in the region of the voltage drop in the constriction. On the other hand, the DFT-NEGF forces in the "bulk" part of the constriction are $\sim 0.05 \mathrm{nN}$, and thus a factor of 4 smaller than ballistic-bulk, while the ratio of the currents at this voltage is, however, roughly a factor of 20. So it is clear that the quasigap in the transmission seen in Fig. 2(a) due to the connection to the graphene electrode is important.

\section{Simple picture}

The extended Hückel Theory [39] can give some insight into the relation we observe between the change in bond forces and overlap populations. In this approach the Hamiltonian is proportional to the overlap matrix of atomic Slater type orbitals. In a homogeneous system involving mainly one orbital on each atom, such as the $\pi$ system in our case, we have $\mathbf{H} \propto \mathbf{S}$. For a roughly exponential dependence of overlap matrix elements on distance one may have the bond-length derivative, $d \mathbf{S} / d b \propto \mathbf{1}-\mathbf{S}, b=\left|\vec{r}_{i j}\right|$. Thus one may for $i, j$ being neighboring atoms approximately have $d \mathbf{H}_{i j} / d b \propto \mathbf{S}_{i j}$ relating the change in bond force, Eq. (6), with the change in overlap population, Eq. (9). Clearly, there will be differences in the on-site potentials, $\mathbf{H}_{i i}$, on atoms, e.g., in the presence of the voltage drop, or surface atoms bonded to H. However, as we see in Figs. 4(b), 4(e) and 7(c), the rough proportionality still holds.

\section{SUMMARY}

Summing up, we have analyzed nonequilibrium forces due to the presence of current in graphene nanoconstrictions by employing first principles transport calculations. We have shown that the induced forces are related to a rearrangement of bond charges due to left/right incoming scattering states. The forces and charges are maximal in the region where the potential drop takes place, because scattering happens there and dipoles are induced. We have further demonstrated forces that exist without potential drop and can thus be considered as purely "current-induced".

Our theoretical work can help our understanding of current-induced strains, bond-breaking processes [40,41], and mechanisms that lead to the destruction of devices at the atomic scale.

\section{COMPUTATIONAL DETAILS: DFT PARAMETERS}

The calculations were done using the SIESTA/TRANSIESTA code with the PBE-GGA functional for exchange correlation and a SZP basis set [42]. Spin polarization is not considered. 
The mesh cutoff was 300 Ry. In SIESTA we used an optimized $k$-point sampling according to the bias window. The transport calculations were averaged over 25 to 50 transverse $k$ points. In the bulk calculations 1000 points along the ribbon are used.

Physical quantities like transmission, current, overlap population, and COOP were extracted using TBtrans and SISL [43].

\section{ACKNOWLEDGMENTS}

We thank Tue Gunst and Gaetano Calogero for helpful discussions. Funding by Villum Fonden (Grant No. 00013340) and the Danish Research Foundation (Project DNRF103) for the Center for Nanostructured Graphene $(\mathrm{CNG})$ is acknowledged.
[1] C. Sabater, C. Untiedt, and J. M. van Ruitenbeek, Beilstein J. Nanotechnol. 6, 2338 (2015).

[2] J. Moser, A. Barreiro, and A. Bachtold, Appl. Phys. Lett. 91, 163513 (2007).

[3] C. Schirm, M. Matt, F. Pauly, J. C. Cuevas, P. Nielaba, and E. Scheer, Nat. Nanotechnol. 8, 645 (2013).

[4] A. K. Geim and K. S. Novoselov, Nat. Mater. 6, 183 (2007).

[5] H. Sadeghi, J. A. Mol, C. S. Lau, G. A. D. Briggs, J. Warner, and C. J. Lambert, Proc. Natl. Acad. Sci. USA 112, 2658 (2015).

[6] F. Prins, A. Barreiro, J. W. Ruitenberg, J. S. Seldenthuis, N. Aliaga-Alcalde, L. M. K. Vandersypen, and H. S. J. van der Zant, Nano Lett. 11, 4607 (2011).

[7] K. Ullmann, P. B. Coto, S. Leitherer, A. Molina-Ontoria, N. Martin, M. Thoss, and H. B. Weber, Nano Lett. 15, 3512 (2015).

[8] S. Leitherer, P. B. Coto, K. Ullmann, H. B. Weber, and M. Thoss, Nanoscale 9, 7217 (2017).

[9] H. Sun, Z. Jiang, N. Xin, X. Guo, S. Hou, and J. Liao, ChemPhysChem 19, 2258 (2018).

[10] H. Zhang, W. Bao, Z. Zhao, J.-W. Huang, B. Standley, G. Liu, F. Wang, P. Kratz, L. Jing, M. Bockrath, and C. N. Lau, Nano Lett. 12, 1772 (2012).

[11] B. Standley, W. Bao, H. Zhang, J. Bruck, C. N. Lau, and M. Bockrath, Nano Lett. 8, 3345 (2008).

[12] P. J. Harris, Carbon 122, 504 (2017).

[13] X. Jia, M. Hofmann, V. Meunier, B. G. Sumpter, J. CamposDelgado, J. M. J. M. Romo-Herrera, H. Son, Y.-P. Hsieh, A. Reina, J. Kong, M. Terrones, and M. S. Dresselhaus, Science 323, 1701 (2009).

[14] A. Barreiro, F. Börrnert, M. H. Rümmeli, B. Büchner, and L. M. K. Vandersypen, Nano Lett. 12, 1873 (2012).

[15] Z. J. Qi, C. Daniels, S. J. Hong, Y. W. Park, V. Meunier, M. Drndic, and A. T. C. Johnson, ACS Nano 9, 3510 (2015).

[16] C. Daniels, Z. Bullard, E. C. Girao, and V. Meunier, Carbon 78, 196 (2014).

[17] D. Dundas, E. J. McEniry, and T. N. Todorov, Nat. Nanotechnol. 4, 99 (2009).

[18] M. Di Ventra, S. T. Pantelides, and N. D. Lang, Phys. Rev. Lett. 88, 046801 (2002).

[19] J.-T. Lü, R. B. Christensen, J.-S. Wang, P. Hedegård, and M. Brandbyge, Phys. Rev. Lett. 114, 096801 (2015).

[20] V. M. García-Suárez, A. García-Fuente, D. J. Carrascal, E. Burzurí, M. Koole, H. S. J. van der Zant, M. El Abbassi, M. Calame, and J. Ferrer, Nanoscale 10, 18169 (2018).
[21] P. Gehring, H. Sadeghi, S. Sangtarash, C. S. Lau, J. Liu, A. Ardavan, J. H. Warner, C. J. Lambert, G. A. D. Briggs, and J. A. Mol, Nano Lett. 16, 4210 (2016).

[22] F. Börrnert, A. Barreiro, D. Wolf, M. I. Katsnelson, B. Büchner, L. M. K. Vandersypen, and M. H. Rümmeli, Nano Lett. 12, 4455 (2012).

[23] T. Gunst, J.-T. Lü, P. Hedegård, and M. Brandbyge, Phys. Rev. B 88, 161401 (2013).

[24] T. Gunst, T. Markussen, K. Stokbro, and M. Brandbyge, Phys. Rev. B 93, 245415 (2016).

[25] N. Papior, T. Gunst, D. Stradi, and M. Brandbyge, Phys. Chem. Chem. Phys. 18, 1025 (2016).

[26] G. Z. Magda, X. Jin, I. Hagymási, P. Vancsó, Z. Osváth, P. Nemes-Incze, C. Hwang, L. P. Biró, and L. Tapasztó, Nature (London) 514, 608 (2014).

[27] D. S. Fisher and P. A. Lee, Phys. Rev. B 23, 6851 (1981).

[28] M. Büttiker, Y. Imry, R. Landauer, and S. Pinhas, Phys. Rev. B 31, 6207 (1985).

[29] S. Nakanishi and M. Tsukada, Phys. Rev. Lett. 87, 126801 (2001).

[30] T. N. Todorov, J. Phys.: Condens. Matter 14, 3049 (2002).

[31] G. Solomon, C. Herrmann, T. Hansen, V. Mujica, and M. Ratner, Nat. Chem. 2, 223 (2010).

[32] M. Brandbyge, J.-L. Mozos, P. Ordejón, J. Taylor, and K. Stokbro, Phys. Rev. B 65, 165401 (2002).

[33] M. Ternes, C. González, C. P. Lutz, P. Hapala, F. J. Giessibl, P. Jelínek, and A. J. Heinrich, Phys. Rev. Lett. 106, 016802 (2011).

[34] M. Brandbyge, K. Stokbro, J. Taylor, J.-L. Mozos, and P. Ordejón, Phys. Rev. B 67, 193104 (2003).

[35] R. S. Mulliken, J. Chem. Phys. 23, 1833 (1955).

[36] N. Asoudegi, M. Soleimani, and M. Pourfath, J. Appl. Phys 125, 144503 (2019).

[37] R. Hoffmann, Rev. Mod. Phys. 60, 601 (1988).

[38] R. Landauer, IBM J. Res. Dev. 1, 223 (1957).

[39] R. Hoffmann, J. Chem. Phys. 39, 1397 (1963).

[40] T. N. Todorov, J. Hoekstra, and A. P. Sutton, Phys. Rev. Lett. 86, 3606 (2001).

[41] A. Erpenbeck, C. Schinabeck, U. Peskin, and M. Thoss, Phys. Rev. B 97, 235452 (2018).

[42] N. Papior, N. Lorente, T. Frederiksen, A. García, and M. Brandbyge, Comput. Phys. Commun. 212, 8 (2017).

[43] N. Papior, sisl: v0.9.4, 2018; doi:10.5281/zenodo.597181. 